\title{
ON LOWER BOUNDS FOR THE KIRCHHOFF INDEX
}

\author{
I. Ž. Milovanović, E. I. Milovanović \\ Faculty of Electronic Engineering, \\ Beogradska 14, P.O.Box 73, 18000 Niš, Serbia \\ e-mail: $\{$ igor, ema\}@elfak.ni.ac.rs
}

(Received December 4, 2016)

ABSTRACT. Let $G$ be a simple graph of order $n \geq 2$ with $m$ edges. Denote by $d_{1} \geq d_{2} \geq$ $\cdots \geq d_{n}>0$ the sequence of vertex degrees and by $\mu_{1} \geq \mu_{2} \geq \cdots \geq \mu_{n-1}>\mu_{n}=0$ the Laplacian eigenvalues of the graph $G$. Lower bounds for the Kirchhoff index, $K f(G)=$ $n \sum_{i=1}^{n-1} \frac{1}{\mu_{i}}$, are obtained.

\section{Introduction}

Let $G=(V, E), V=\{1,2, \ldots, n\}, E=\left\{e_{1}, e_{2}, \ldots, e_{m}\right\}$ be a simple connected graph of order $n \geq 3$ and size $m$. If vertices $i$ and $j$ are adjacent, we denote it as $i \sim j$. Denote by $d_{1} \geq d_{2} \geq \cdots \geq d_{n}>0$ a sequence of vertex degrees, and by $\Delta$ and $\delta$ the greatest and the smallest vertex degrees, respectively. Let $\mathbf{A}$ be the adjacency matrix of $G$, and $\mathbf{D}=\operatorname{diag}\left(d_{1}, d_{2}, \ldots, d_{n}\right)$ the diagonal matrix of its vertex degrees. Then $\mathbf{L}=\mathbf{D}-\mathbf{A}$ is the Laplacian matrix of $G$. Eigenvalues of $\mathbf{L}$, $\mu_{1} \geq \mu_{2} \geq \cdots \geq \mu_{n-1}>\mu_{n}=0$, are the Laplacian eigenvalues of graph $G$.

Some well-known properties of the Laplacian eigenvalues are (see for example [3]):

$$
\sum_{i=1}^{n-1} \mu_{i}=\sum_{i=1}^{n} d_{i}=2 m \quad \text { and } \quad \sum_{i=1}^{n-1} \mu_{i}^{2}=\sum_{i=1}^{n} d_{i}^{2}+\sum_{i=1}^{n} d_{i}=M_{1}+2 m
$$


where

$$
M_{1}=M_{1}(G)=\sum_{i=1}^{n} d_{i}^{2}=\sum_{i \sim j}\left(d_{i}+d_{j}\right)=\sum_{i=1}^{m}\left(d\left(e_{i}\right)+2\right)
$$

is the first Zagreb index introduced in [11]. In the same paper the second Zagreb index, $M_{2}$, and so called forgotten index, $F$, were defined as

$$
M_{2}=M_{2}(G)=\sum_{i \sim j} d_{i} d_{j} \quad \text { and } \quad F=F(G)=\sum_{i=1}^{n} d_{i}^{3}=\sum_{i \sim j}\left(d_{i}^{2}+d_{j}^{2}\right) .
$$

More on the invariant $F$ one can find in $[7,9]$.

Matrix $\mathbf{L}^{*}=\mathbf{D}^{-1 / 2} \mathbf{L} \mathbf{D}^{-1 / 2}=\mathbf{I}-\mathbf{D}^{-1 / 2} \mathbf{A} \mathbf{D}^{-1 / 2}$ is the normalized Laplacian matrix of $G$. Its eigenvalues, $\rho_{1} \geq \rho_{2} \geq \cdots \geq \rho_{n-1}>\rho_{n}=0$, represent normalized Laplacian eigenvalues of $G$. The following is valid for $\rho_{i}, i=1,2, \ldots, n$, (see [3]):

$$
\sum_{i=1}^{n-1} \rho_{i}=n \quad \text { and } \quad \sum_{i=1}^{n-1} \rho_{i}^{2}=n+2 R_{-1},
$$

where

$$
R_{-1}=\sum_{i \sim j} \frac{1}{d_{i} d_{j}}
$$

is the general Randić index (also called branching index) introduced in [27].

The Kirchhoff index of a connected graph is defined as (see [14]):

$$
K f(G)=\sum_{i<j} r_{i j}
$$

where $r_{i j}$ is the effective resistance distance between vertices $i$ and $j$. The following more appropriate formula from application point of view was put forward in [10]

$$
K f(G)=n \sum_{i=1}^{n-1} \frac{1}{\mu_{i}}
$$

This, in turn, triggered the study of this invariant and its applications in various areas, including spectral graph theory, molecular chemistry, computer science, etc. (see for example $[7,9-11,14,18,27])$.

Before we proceed, let us define one special class of $d$-regular graphs $\Gamma_{d}$ (see [25]). Let $N(i)$ be a set of all neighborhoods of the vertex $i$, i.e. $N(i)=\{k \mid k \in V, k \sim i\}$, and $d(i, j)$ the distance between vertices $i$ and $j$. Denote by $\Gamma_{d}$ a set of all $d$-regular 
graphs, $1 \leq d \leq n-1$, with diameter $D=2$ and $|N(i) \cap N(j)|=d$. Further, denote by $t=t(G)$ a number of spanning trees of the connected graph

$$
t=t(G)=\frac{1}{n} \prod_{i=1}^{n-1} \mu_{i}
$$

and by $I D=I D(G)$ the graph invariant called inverse degree

$$
I D=I D(G)=\sum_{i=1}^{n} \frac{1}{d_{i}}
$$

In this paper we are concerned with the lower bounds of $K f(G)$ which depend on some of the parameters $n, m, \Delta$, and invariants $R_{-1}, M_{1}, M_{2}$ or $F$. Before going further, we recall some results from the literature needed for our subsequent consideration.

\section{Preliminaries}

In this section we outline some results for the invariants $K f(G), M_{1}, M_{2}, F, t$ and $R_{-1}$ that will be needed in the remainder of the paper.

In [28] the following result was proved for the $K f(G)$ :

Lemma 2.1. [28] Let $G$ be a simple connected graph with $n \geq 2$ vertices and $m$ edges. Then

$$
K f(G) \geq-1+(n-1) \sum_{i=1}^{n} \frac{1}{d_{i}},
$$

with equality if and only if $G \cong K_{n}$ or $G \cong K_{r, n-r}, 1 \leq r \leq\left\lfloor\frac{n}{2}\right\rfloor$.

Remark 2.2. We believe that equality in (1) holds also when $G \in \Gamma_{d}$ and $G \cong K_{n}-e$.

This only increases importance of the above inequality.

In [23] the following was proved for the general Randic index:

Lemma 2.3. [23] Let $G$ be a simple connected graph with $n \geq 3$ vertices and $m$ edges. Then, for any real $k$ with the property $\rho_{1} \geq k \geq \rho_{n-1}$, holds

$$
2 R_{-1} \geq \frac{n}{n-1}+\frac{n-1}{n-2}\left(k-\frac{n}{n-1}\right)^{2},
$$

with equality if and only if $k=\frac{n}{n-1}$ and $G \cong K_{n}$, or $k=2$ and $G \cong K_{\frac{n}{2}}, \frac{n}{2}$. 
In $[13,22,24]$ for the Forgotten index the following results were established:

Lemma 2.4. [13] Let $G$ be a simple graph with $n$ vertices and $m$ edges. Then

$$
F \leq(\Delta+\delta) M_{1}-2 m \Delta \delta
$$

with equality if and only if $G$ is regular or bidegreed graph.

Lemma 2.5. [24] Let $G$ be a simple connected graph with $n \geq 2$ vertices and $m$ edges. Then

$$
F \leq 2 m\left(\Delta^{2}+\Delta \delta+\delta^{2}\right)-n \Delta \delta(\Delta+\delta)
$$

with equality if and only if $G$ is regular or bidegreed graph.

Lemma 2.6. [22] Let $G$ be a simple connected graph with $n \geq 2$ vertices and $m$ edges. Then

$$
F \leq \frac{M_{1}}{2 m}+2 m \beta(S)(\Delta-\delta)^{2}
$$

where

$$
\beta(S)=\frac{1}{2 m} \sum_{i \in S} d_{i}\left(1-\frac{1}{2 m} \sum_{i \in S} d_{i}\right)
$$

and $S$ is a subset of $I=\{1,2, \ldots, n\}$ which minimizes the expression

$$
\left|\sum_{i \in S} d_{i}-m\right|
$$

Equality in (5) holds if and only if $L(G)$ is regular.

In [4] (see also [15]) for the first Zagreb index, $M_{1}$, the following was proved:

Lemma 2.7. [4] Let $G$ be a simple connected graph with $n \geq 2$ vertices and $m$ edges. Then

$$
M_{1} \leq 2(\Delta+\delta) m-n \Delta \delta
$$

with equality if and only if $G$ is regular or bidegreed graph.

For the same invariant in [21] the following was proved:

Lemma 2.8. [21] Let $G$ be a simple connected graph with $n \geq 2$ vertices and $m$ edges. Then

$$
M_{1} \leq \frac{4 m^{2}}{n}+n \alpha(n)(\Delta-\delta)^{2}
$$


where

$$
\alpha(n)=\frac{1}{4}\left(1-\frac{(-1)^{m+1}+1}{2 n^{2}}\right) .
$$

Equality in (7) holds if and only if $G$ is regular.

For the number of spanning trees, $t$, of a graph the following was proved in [5]:

Lemma 2.9. [5] Let $G$ be a simple connected graph with $n \geq 2$ vertices and $m$ edges. Then

$$
t \leq \frac{1}{n}\left(\frac{4 m^{2}-M_{1}-2 m}{(n-1)(n-2)}\right)^{\frac{n-1}{2}},
$$

with equality if and only if $G \cong K_{n}$.

For the same invariant in [1] the following was proved:

Lemma 2.10. [1] Let $G$ be a simple connected graph with $n \geq 2$ vertices and $m$ edges. Then

$$
t \geq\left(\frac{\prod_{i=1}^{n} d_{i}}{2 m}\right)\left(\frac{1}{n-1}\left(n^{2}-(n-2)\left(n+2 R_{-1}\right)\right)\right)^{\frac{n-1}{2}}
$$

with equality if and only if $G \cong K_{n}$.

\section{Main results}

We will first prove one general result for the lower bounds of $K f(G)$ in terms of one of the invariants $R_{-1}, M_{2}, F$ or $M_{1}$.

Theorem 3.1. Let $G$ be a simple connected graph with $n \geq 2$ vertices and $m$ edges. Then

$$
\begin{aligned}
& K f(G) \geq-1+2(n-1) R_{-1}, \\
& K f(G) \geq-1+\frac{2(n-1) m^{2}}{M_{2}}, \\
& K f(G) \geq-1+\frac{4(n-1) m^{2}}{F}, \\
& K f(G) \geq-1+\frac{4(n-1) m^{2}}{\Delta M_{1}} .
\end{aligned}
$$

Equalities hold if and only if $G \cong K_{n}$, or $G \cong K_{\frac{n}{2}, \frac{n}{2}}$, or $G \in \Gamma_{d}$. 
Proof. In [19] the following inequality was proved

$$
I D \geq 2 R_{-1}
$$

From the inequality

$$
\sum_{i \sim j} d_{i} d_{j} \sum_{i \sim j} \frac{1}{d_{i} d_{j}} \geq m^{2}
$$

follows that

$$
R_{-1} \geq \frac{m^{2}}{M_{2}}
$$

Also, the following holds

$$
2 M_{2}=2 \sum_{i \sim j} d_{i} d_{j} \leq \sum_{i \sim j}\left(d_{i}^{2}+d_{j}^{2}\right)=\sum_{i=1}^{n} d_{i}^{3}=F
$$

and

$$
F_{1}=\sum_{i=1}^{n} d_{i}^{3} \leq \Delta \sum_{i=1}^{n} d_{i}^{2}=\Delta M_{1}
$$

Accordingly, we have that

$$
I D \geq 2 R_{-1} \geq \frac{2 m^{2}}{M_{2}} \geq \frac{4 m^{2}}{F} \geq \frac{4 m^{2}}{\Delta M_{1}} .
$$

From (14) and (1) inequalities (10) - (13) are obtained.

If in $(10)-(13)$ invariants $R_{-1}, M_{2}, F$ and $M_{1}$ are replaced with corresponding lower bounds, a number of lower bounds for $K f(G)$ depending on various graph parameters can be obtained. In what follows we will illustrate this.

From (10) and (2) the following corollary of Theorem 3.1 is obtained.

Corollary 3.2. Let $G$ be a simple connected graph with $n \geq 3$ vertices and $m$ edges. Then for any real $k, \rho_{1} \geq k \geq \rho_{n-1}$, holds

$$
K f(G) \geq n-1+\frac{(n-1)^{2}}{n-2}\left(k-\frac{n}{n-1}\right)^{2},
$$

with equality if and only if $k=\frac{n}{n-1}$ and $G \cong K_{n}$, or $k=2$ and $G \cong K_{\frac{n}{2}, \frac{n}{2}}$.

Since

$$
\rho_{1} \geq \frac{\Delta+1}{\Delta} \geq \frac{n}{n-1} \geq \rho_{n-1},
$$

according to (15), the following corollary of Theorem 3.1 holds. 
Corollary 3.3. Let $G$ be a simple connected graph with $n \geq 3$ vertices and $m$ edges.

Then

$$
K f(G) \geq n-1+\frac{(n-1)^{2}}{n-2} \max \left\{\left(\rho_{1}-\frac{n}{n-1}\right)^{2},\left(\rho_{n-1}-\frac{n}{n-1}\right)^{2}\right\},
$$

with equality if and only if $G \cong K_{n}$ or $G \cong K_{\frac{n}{2}, \frac{n}{2}}$.

Corollary 3.4. Let $G$ be a simple connected graph with $n \geq 2$ vertices and $m$ edges. Then

$$
K f(G) \geq n-1
$$

with equality if and only if $G \cong K_{n}$.

The inequality (16) was proved in [17]. It is not difficult to see that (16) can be obtained from (10) and inequality (see [16])

$$
2 R_{-1} \geq \frac{n}{n-1}
$$

Corollary 3.5. Let $G$ be a simple connected graph with $n \geq 3$ vertices and $m$ edges.

Then

$$
K f(G) \geq n-1+\frac{(n-1-\Delta)^{2}}{(n-2) \Delta^{2}}
$$

with equality if and only if $G \cong K_{n}$.

Corollary 3.6. Let $G$ be a simple connected graph with $n \geq 2$ vertices and $m$ edges. Then

$$
K f(G) \geq \frac{n(n-1)-\Delta}{\Delta}
$$

with equality if and only if $G \cong K_{n}$, or $G \cong K_{\frac{n}{2}, \frac{n}{2}}$, or $G \in \Gamma_{d}$.

Proof. The inequality (17) is obtained from (10) and inequality

$$
R_{-1} \geq \frac{n}{2 \Delta}
$$

which proved in [2].

The inequality (17) was proved in [25].

According to Lemma 2.4 the following corollary of Theorem 3.1 can be obtained. 
Corollary 3.7. Let $G$ be a simple connected graph with $n \geq 2$ vertices and $m$ edges.

Then

$$
K f(G) \geq \frac{4(n-1) m^{2}}{(\Delta+\delta) M_{1}-2 m \delta \Delta}-1
$$

with equality if and only if $G \cong K_{n}$, or $G \cong K_{\frac{n}{2}}, \frac{n}{2}$, or $G \in \Gamma_{d}$.

Corollary 3.8. Let $G$ be a simple connected graph with $n \geq 2$ vertices and $m$ edges.

Then

$$
K f(G) \geq \frac{32(n-1) m^{3} \delta \Delta}{(\Delta+\delta)^{2} M_{1}^{2}}-1
$$

with equality if and only if $G \cong K_{n}$, or $G \cong K_{\frac{n}{2}, \frac{n}{2}}$, or $G \in \Gamma_{d}$.

Proof. After applying the arithmetic-geometric mean (AG) inequality on (3), i.e. on

$$
F+2 m \Delta \delta \leq(\Delta+\delta) M_{1}
$$

the inequality

$$
F \leq \frac{(\Delta+\delta)^{2} M_{1}^{2}}{8 m \delta \Delta}
$$

is obtained. From this and (12) we obtain (18).

From Lemma 2.5 the following corollary of Theorem 3.1 is obtained.

Corollary 3.9. Let $G$ be a simple connected graph with $n \geq 2$ vertices and $m$ edges. Then

$$
K f(G) \geq \frac{4(n-1) m^{2}}{2 m\left(\Delta^{2}+\Delta \delta+\delta^{2}\right)-n \Delta \delta(\Delta+\delta)}-1,
$$

with equality if and only if $G \cong K_{n}$, or $G \cong K_{\frac{n}{2}, \frac{n}{2}}$, or $G \in \Gamma_{d}$.

Similarly, from Lemma 5 and (12) the following corollary of Theorem 3.1 is obtained.

Corollary 3.10. Let $G$ be a simple connected graph with $n \geq 2$ vertices and $m$ edges.

Then

$$
K f(G) \geq \frac{8(n-1) m^{3}}{M_{1}^{2}+4 m^{2} \beta(S)(\Delta-\delta)^{2}}-1,
$$

where

$$
\beta(S)=\frac{1}{2 m} \sum_{i \in S} d_{i}\left(1-\frac{1}{2 m} \sum_{i \in S} d_{i}\right)
$$


and $S$ is a subset of $I=\{1,2, \ldots, n\}$ which minimizes the expression

$$
\left|\sum_{i \in S} d_{i}-m\right|
$$

Equality holds if and only if $G \cong K_{n}$, or $G \cong K_{\frac{n}{2}, \frac{n}{2}}$, or $G \in \Gamma_{d}$.

Corollary 3.11. Let $G$ be a simple connected graph with $n \geq 2$ vertices and $m$ edges. Then

$$
K f(G) \geq \frac{4(n-1) m^{2}}{\Delta(2 m(\Delta+\delta)-n \Delta \delta)}-1,
$$

with equality if and only if $G \cong K_{n}$, or $G \cong K_{\frac{n}{2}, \frac{n}{2}}$, or $G \in \Gamma_{d}$.

Proof. The required inequality is obtained from (6) and (13).

Corollary 3.12. Let $G$ be a simple connected graph with $n \geq 2$ vertices and $m$ edges.

Then

$$
K f(G) \geq \frac{4 n(n-1) \delta}{(\Delta+\delta)^{2}}-1,
$$

with equality if and only if $G \cong K_{n}$, or $G \cong K_{\frac{n}{2}, \frac{n}{2}}$, or $G \in \Gamma_{d}$.

Proof. After applying the AG inequality on (6), i.e. on

$$
M_{1}+n \Delta \delta \leq 2 m(\Delta+\delta)
$$

the inequality

$$
M_{1} \leq \frac{(\Delta+\delta)^{2} m^{2}}{n \Delta \delta}
$$

is obtained (see $[6,8,12,20]$ ). The required inequality is obtained from the above inequality and (13).

From (7) and (13) the following corollary of Theorem 3.1 is obtained.

Corollary 3.13. Let $G$ be a simple connected graph with $n \geq 2$ vertices and $m$ edges. Then

$$
K f(G) \geq \frac{4 n(n-1) m^{2}}{\Delta\left(4 m^{2}+n^{2} \alpha(n)(\Delta-\delta)^{2}\right)}-1
$$

where

$$
\alpha(n)=\frac{1}{4}\left(1-\frac{(-1)^{n+1}+1}{2 n^{2}}\right) .
$$

Equality holds if and only if $G \cong K_{n}$, or $G \cong K_{\frac{n}{2}, \frac{n}{2}}$, or $G \in \Gamma_{d}$. 
Corollary 3.14. Let $G$ be a simple connected graph with $n \geq 2$ vertices and $m$ edges.

Then

$$
K f(G) \geq \frac{2 m(n-1)}{\Delta^{2}}-1,
$$

with equality if and only if $G \cong K_{n}$, or $G \cong K_{\frac{n}{2}}, \frac{n}{2}$, or $G \in \Gamma_{d}$.

Proof. The required result is obtained from (13) and inequality

$$
M_{1} \leq 2 m \Delta
$$

The following corollary of Theorem 3.1 sets up a lower bound for $K f(G)$ in terms of parameters $n$ and $m$ and the invariant $t$.

Corollary 3.15. Let $G$ be a simple connected graph with $n \geq 3$ vertices and $m$ edges. Then

$$
K f(G) \geq \frac{4(n-1) m^{2}}{\Delta\left(4 m^{2}-2 m-(n-1)(n-2)(n t)^{\frac{2}{n-1}}\right)}-1,
$$

with equality if and only if $G \cong K_{n}$.

Proof. From inequality (8) follows

$$
M_{1} \leq 4 m^{2}-2 m-(n-1)(n-2)(n t)^{\frac{2}{n-1}} .
$$

From the above and inequality (13) we arrive at (19).

Similarly, the following can be proved:

Corollary 3.16. Let $G$ be a simple connected graph with $n \geq 3$ vertices and $m$ edges, and let $t$ be the total number of spanning trees of $G$. Then

$$
K f(G) \geq \frac{n-1}{n-2}\left(2 n-(n-1)\left(\frac{2 m t}{\prod_{i=1}^{n} d_{i}}\right)^{\frac{2}{n-1}}\right)-1,
$$

with equality if and only if $G \cong K_{n}$.

Proof. From (9) follows

$$
2 R_{-1} \geq \frac{1}{n-2}\left(2 n-(n-1)\left(\frac{2 m t}{\prod_{i=1}^{n} d_{i}}\right)^{\frac{2}{n-1}}\right) .
$$

From the above and inequality (10) we obtain the required result. 
Let us note that the connectivity condition for the graph $G$ does not deteriorate the generality of the results. Namely, a graph $G$ can be observed as a union of connected components as well.

Acknowledgement This work was supported by the Serbian Ministry for Education, Science and Technological development.

\section{REFERENCES}

[1] S. B. Bozkurt, D. Bozkurt, On the number of spanning trees of graphs, Sci. World J. (2014) Article ID294038.

[2] M. Cavers, The normalized Laplacian matrix and general Randić index of graphs, Ph. Dissertation, Univ. Regina, Regina, 2010.

[3] F. R. K. Chung, Spectral graph theory, Am. Math. Soc., Providence, 1997.

[4] K. Ch. Das, Maximizing the sum of squares of the degrees of a graph, Discr. Math. 285 (2004) 57-66.

[5] K. C. Das, A. S. Cevik, I. N. Cangul, The number of spanning trees of a graph, J. Ineq. Appl. 2013 (2013) 1-13.

[6] G. H. Fath - Tabar, Old and new Zagreb indices of graphs, MATCH Commun. Math. Comput. Chem. 65 (2011) 79-84.

[7] B. Furtula, I. Gutman, A forgotten topological index, J. Math. Chem. 53 (2015) $1184-1190$.

[8] T. Guixian, H. Tinzhy, C. Shuyu, Bounds on the algebraic connectivity of graphs, Advanc. Math. 41 (2) (2012) 217-224.

[9] I. Gutman, K. C. Das, The first Zagreb index 30 years after, MATCH Commun. Math. Comput. Chem. 50 (2004) 83-92.

[10] I. Gutman, B. Mohar, The quasi-Wiener and Kirchhoff indices coincide, J. Chem. Inf. Comput. Sci. 36 (1996) 982-985. 
[11] I. Gutman, N. Trinajstić, Graph theory and molecular orbitas. Total $\pi$-electron energy of alternant hydrocarbons, Chem. Phys. Lett. 17 (1972) 535-538.

[12] A. Ilić, M. Ilić, B. Liu, On the upper bounds for the first Zagreb index, Kragujevac J. Math. 32 (1) (2011) 173-182.

[13] A. Ilić, B. Zhou, On reformulated Zagreb indices, Discr. Appl. Math. 160 (2012) $204-209$.

[14] D. J. Klein, M. Randić, Resistance distance, J. Math. Chem. 12 (1993) 81-95.

[15] J. Li, W. C. Shiu, A. Chang, On the Laplacian Estrada index of graphs, Appl. Anal. Discr. Math. 13 (2009) 147-156.

[16] X. Li, Y. Yang, Sharp bounds for the general Randić index, MATCH Commun. Math. Comput. Chem. 51 (2004) 155-166.

[17] I. Lukovits, S. Nikolić, N. Trinajstić, Resistance distance in regular graphs, Int. J. Quantum Chem. 71 (3) (1999) 217-225.

[18] J. Liu, J. Cao, X. F. Pan, A. Elaiw, The Kirchhoff index of hypercubes and related complex networks, Discr. Dynam. Natur. Sci. (2013) Article ID543189.

[19] M. Liu, H. Liu, F. Tian, The connectivity index, MATCH Commun. Math. Comput. Chem. 51 (2004) 149-154.

[20] M. Liu, B. Liu, New sharp upper bounds for the first Zagreb index, MATCH Commun. Math. Comput. Chem. 62 (2009) 689-698.

[21] E. I. Milovanović, I. Ž. Milovanović, Sharp bounds for the first Zagreb index and first Zagreb coindex, Miskolc Math. Notes. 16 (2) (2015) 1017-1024.

[22] E. I. Milovanović, I. Ž. Milovanović, E. Ć. Dolićanin, E. Glogić, A note on the first reformulated Zagreb index, Appl. Math. Comput. 273 (2016) 16-20.

[23] I. Ž. Milovanović, E. I. Milovanović, Remarks on lower bounds on the general Randić index $R_{-1}$ of graph, Appl. Math. Comput. Sci. 1 (1) (2016) 9-13. 
[24] I. Ž. Milovanović, V. M. Ćirić, I. Z. Milentijević, E. I. Milovanović, On some spectral, vertex and edge degree-based graph invariants, MATCH Commun. Math. Comput. Chem. 71 (1)(2017) 177-188.

[25] J. L. Palacios, Some additional bounds for the Kirchhoff index, MATCH Commun. Math. Comput. Chem. 75 (2016) 365-372.

[26] J. L. Palacios, J. M. Renom, Broder and Karlin's formula for lifting times and the Kirchhoff index, Int. J. Quantum Chem. 111 (2011) 35-39.

[27] M. Randić, On characterization of molecular branching, J. Amer. Chem. Soc. 97 (1975) 6609-6615

[28] B. Zhou, N. Trinajstić, A note on Kirchhoff index, Chem. Phys. Lett. 455 (2008) 611-619. 